\title{
Universal amplitude ratios in the three-dimensional Ising universality class
}

\author{
Martin Hasenbusch* \\ Institut für Physik, Humboldt-Universität zu Berlin, \\ Newtonstr. 15, 12489 Berlin, Germany
}

(Dated: October 30, 2018)

\begin{abstract}
We compute a number of universal amplitude ratios in the three-dimensional Ising universality class. To this end, we perform Monte Carlo simulations of the improved Blume-Capel model on the simple cubic lattice. For example, we obtain $A_{+} / A_{-}=0.536(2)$ and $C_{+} / C_{-}=4.713(7)$, where $A_{ \pm}$and $C_{ \pm}$are the amplitudes of the specific heat and the magnetic susceptibility, respectively. The subscripts + and - indicate the high and the low temperature phase, respectively. We compare our results with those obtained from previous Monte Carlo simulations, high and low temperature series expansions, field theoretic methods and experiments.
\end{abstract}

PACS numbers: 05.50.+q, 05.70.Jk, 64.60.F-

*Martin.Hasenbusch@physik.hu-berlin.de 


\section{INTRODUCTION}

In the neighborhood of a second order phase transition various quantities diverge, following power laws. For example, in a magnetic system, the correlation length $\xi$, the magnetic susceptibility $\chi$ and the specific heat $C$ behave as

$$
\xi \simeq f_{ \pm}|t|^{-\nu} \quad, \quad \chi \simeq C_{ \pm}|t|^{-\gamma} \quad, \quad C \simeq A_{ \pm}|t|^{-\alpha},
$$

where $t=\left(T-T_{c}\right) / T_{c}$ is the reduced temperature. The symbol $\simeq$ means asymptotically equal; corrections vanish as $t \rightarrow 0$. Critical exponents like $\nu, \gamma$ and $\alpha$ are universal. That means, they take exactly the same value for all systems in a given universality class. A universality class is characterized by the spacial dimension of the system, the range of the interaction and the symmetry of the order parameter. For reviews on critical phenomena and its modern theory, the renormalization group see for example [1 4 ] .

While individual amplitudes like $f_{+}, f_{-}, C_{+}, C_{-}, A_{+}$and $A_{-}$depend on the details of the system, amplitude ratios like $f_{+} / f_{-}, C_{+} / C_{-}$and $A_{+} / A_{-}$are universal. The indices + and - indicate the high and the low temperature phase, respectively. In addition to these simple ratios, there are also more complicated universal combinations of amplitudes. The combinations of the corresponding quantities are dimensionless. This means that they have a combined critical exponent that is equal to zero. Such amplitude ratios have been determined for a number of experimental systems and computed by using various theoretical approaches like the $\epsilon$-expansion, perturbation theory in three dimensions fixed, high and low temperature series expansions and Monte Carlo simulations. A summary of results is given in refs. [4, 5]. Here we study universal amplitude ratios in the universality class of the three-dimensional Ising model with short range interactions, which is characterized by the $\mathbb{Z}_{2}$ symmetry of the order parameter. This universality class is supposed to be realized in a huge range of experimental systems: binary mixtures, uniaxial magnets or micellar systems; see [4, 5].

At finite values of the reduced temperature, power laws (1) are subject to corrections. For example the magnetic susceptibility behaves as

$$
\chi=C_{ \pm}|t|^{-\gamma}\left(1+a_{ \pm}|t|^{\theta}+b t+\ldots\right),
$$

where $\theta=\nu \omega=0.524(4)[6]$. The amplitudes $C_{ \pm}, a_{ \pm}$and $b$ in general depend on the parameters of the system. Already in 1982 the authors of ref. [7] have demonstrated that for a model that interpolates between the Gaussian and the Ising model there is one value of the interpolation parameter, where $a_{ \pm}$vanishes. Renormalization group predicts that the zero of leading correction amplitudes is the same for all quantities. In the following we shall call a model with $a_{ \pm}=0$ an improved model. Studying improved models simplifies the accurate determination of amplitude ratios using Monte Carlo simulations or high and

low temperature series expansions. Here we simulate the improved Blume-Capel model on the simple cubic lattice. For the definition of this model see the next section. Our main motivation to perform these simulations was to compute the energy density of the bulk system in a large range of inverse temperatures. This quantity is needed in our ongoing study of the thermal Casimir effect in the three-dimensional Ising universality class. Here we use the data generated for various quantities to update the estimates of a number of universal amplitude ratios. Computing universal amplitude ratios, we follow the strategy of [8, 9], where the spin-1/2 Ising model had been studied and more recently 
[10, 11], where we had studied an improved model in the XY universality class in three dimensions.

Our results are essentially consistent with previous Monte Carlo studies [8, 12, 13] and the most recent analysis of high and low temperature series expansions [14]. Typically we reduce the error bars by a factor of two to three compared with these studies. Estimates obtained by using field theoretic methods are typically by a factor of ten less precise than those obtained here.

The outline of our paper is the following: First we define the model and the observables that we have measured. Next we discuss the update algorithm and give details of our simulations. Using the data obtained, we extract numerical estimates for various universal amplitude ratios. These estimates are compared with those obtained in previous Monte Carlo simulations, from high and low temperature series expansions, field theoretic methods and experiments. Finally we conclude.

\section{MODEL AND OBSERVABLES}

The Blume-Capel model is characterized by the reduced Hamiltonian

$$
H=-\beta \sum_{<x y>} s_{x} s_{y}+D \sum_{x} s_{x}^{2}-h \sum_{x} s_{x}
$$

where the spin might assume the values $s_{x} \in\{-1,0,1\}$. The sites on the simple cubic lattice are denoted by $x=\left(x_{0}, x_{1}, x_{2}\right)$ with $x_{i}=0,1, \ldots, L_{i}-1$. In the following we shall consider lattices with $L=L_{0}=L_{1}=L_{2}$ in the high temperature phase and $L_{0}=2 L$, $L_{1}=L_{2}=L$ lattices in the low temperature phase. Throughout we consider periodic boundary conditions. The first sum in eq. (3) runs over all pairs of nearest neighbor sites $\langle x y\rangle$ on the lattice and $\beta=1 / k_{B} T$ is the inverse temperature. The partition function is given by $Z=\sum_{\{s\}} \exp (-H)$, where the sum runs over all spin configurations. In the following we shall consider a vanishing external field $h=0$. The parameter $D$ controls the density of vacancies $s_{x}=0$. In the limit $D \rightarrow-\infty$ vacancies are completely suppressed and therefore the spin-1/2 Ising model is recovered. In $d \geq 2$ dimensions the model undergoes a continuous phase transition for $-\infty \leq D<D_{t r i}$ at a $\beta_{c}$ that depends on $D$. For $D>D_{t r i}$ the model undergoes a first order phase transition. Refs. [15 17] give for the three-dimensional simple cubic lattice $D_{t r i} \approx 2.006,2.05$ and $D_{t r i}=2.0313(4)$, respectively.

Numerically it has been shown that on the line of second order phase transitions there is a value $D^{*}$ of the parameter $D$, where leading corrections to scaling vanish. In ref. [18] we found $D^{*}=0.641(8)$. One should note that little effort was made to estimate the systematical error due to subleading corrections to scaling. Recently we have determined

$D^{*}=0.656(20)$ [6], where now systematical errors are taken into account. In [6] we have simulated the model at $D=0.641$ and $D=0.655$ in the neighborhood of the critical point. Using a standard finite size scaling analysis we find

$$
\begin{aligned}
& \beta_{c}(0.641)=0.38567122(5) \\
& \beta_{c}(0.655)=0.387721735(25)
\end{aligned}
$$

as estimates of the inverse critical temperature. We also find that the amplitudes of leading corrections at $D=0.655$ are reduced by at least a factor of 30 compared with the spin-1/2 Ising model. 


\section{A. The energy density and the specific heat}

Here, we define the energy density as minus the derivative of the reduced free energy density with respect to $\beta$

$$
E=\frac{1}{V} \frac{\partial}{\partial \beta} \ln Z=\frac{1}{V}\left\langle\sum_{<x y>} s_{x} s_{y}\right\rangle,
$$

where $V=L_{0} L_{1} L_{2}$. The specific heat is the derivative of the energy density with respect to $\beta$. One finds

$$
C=\frac{\partial E}{\partial \beta}=\frac{1}{V}\left[\left\langle\left(\sum_{<x y>} s_{x} s_{y}\right)^{2}\right\rangle-\left\langle\sum_{<x y>} s_{x} s_{y}\right\rangle^{2}\right] .
$$

B. The magnetic susceptibility and the second moment correlation length in the high temperature phase

The magnetic susceptibility $\chi$ and the second moment correlation length $\xi_{2 n d}$ are defined as

$$
\chi=\frac{1}{V}\left\langle\left(\sum_{x} s_{x}\right)^{2}\right\rangle
$$

and

$$
\xi_{2 n d}=\sqrt{\frac{\chi / F-1}{4 \sin ^{2} \pi / L}}
$$

where

$$
F=\frac{1}{V}\left\langle\left|\sum_{x} \exp \left(i \frac{2 \pi x_{k}}{L}\right) s_{x}\right|^{2}\right\rangle
$$

is the Fourier transform of the correlation function at the lowest non-zero momentum.

In our simulations in the high temperature phase, we have measured $F$ for the three directions $k=0,1,2$ and have averaged these three results.

C. The magnetization, the magnetic susceptibility and the correlation length in the low temperature phase

The magnetization in presence of a magnetic field is defined by

$$
m(h, L)=\frac{1}{V}\left\langle\sum_{x} s_{x}\right\rangle
$$

where we assume, for simplicity, a fixed ratio $L_{0} / L$ with $L=L_{1}=L_{2}$. The spontaneous magnetization is then defined as

$$
m(0, \infty)=\lim _{h \searrow 0} \lim _{L \rightarrow \infty} m(h, L)
$$


where first the thermodynamic limit is taken. In a Monte Carlo simulation it is too cumbersome to follow this route. Note that $m(0, L)$ at a finite value of $L$ is however exactly zero for symmetry reasons.

To avoid this problem, Binder and Rauch [19] proposed the following definition:

$$
m_{R M S}(0, L)=\frac{1}{V} \sqrt{\left\langle\left(\sum_{x} s_{x}\right)^{2}\right\rangle} .
$$

Here, following eqs. $(20,21)$ of [20], we use

$$
m_{A B S}(0, L)=\frac{1}{V}\left\langle\left|\sum_{x} s_{x}\right|\right\rangle,
$$

which in the low temperature phase converges faster than $m_{R M S}(0, L)$.

The connected two-point correlation function is given by

$$
G(x, y)=\left\langle s_{x} s_{y}\right\rangle-\left\langle s_{x}\right\rangle\left\langle s_{y}\right\rangle
$$

In the low temperature phase, for $h=0$ we replace eq. (15), using eq. (14), by

$$
\left.G_{\text {low }}(x, y)\right|_{h=0}=\left\langle s_{x} s_{y}\right\rangle-m_{A B S}^{2}(0, L) \text {. }
$$

In order to project to zero-momentum states of the transfermatrix, we consider the correlation function

$$
G(r)=\left\langle S_{0} S_{r}\right\rangle-\left\langle S_{0}\right\rangle\left\langle S_{r}\right\rangle
$$

of time slices

$$
S_{x_{0}}=\frac{1}{\sqrt{L_{1} L_{2}}} \sum_{x_{1}, x_{2}} s_{\left(x_{0}, x_{1}, x_{2}\right)} .
$$

Note that with this normalization, the correlation function has a finite thermodynamic limit as $L_{1}, L_{2} \rightarrow \infty$. In the low temperature phase, for vanishing external field $h=0$ we replace $\left\langle S_{0}\right\rangle\left\langle S_{r}\right\rangle$ by $L_{1} L_{2} m_{A B S}^{2}(0, L)$.

The magnetic susceptibility can be written as

$$
\chi=\sum_{r=-\infty}^{\infty} G(r) .
$$

The effective correlation length is given by

$$
\xi_{\text {eff }}(r)=-1 / \ln \left(\frac{G(r+1)}{G(r)}\right) .
$$

The exponential correlation length is defined as $\xi_{\text {exp }}=\lim _{r \rightarrow \infty} \xi_{\text {eff }}(r)$. Since the transfermatrix is positive and symmetric, $\xi_{\text {eff }}$ approaches $\xi_{\text {exp }}$ monotonically from below. The second moment correlation length is defined by

$$
\xi_{2 n d}^{2}=\frac{\mu_{2}}{2 d \chi}
$$


where $d=3$ is the dimension of the system and

$$
\mu_{2}=d \sum_{r=-\infty}^{\infty} r^{2} G(r)
$$

Note that in the thermodynamic limit, the definitions (921) become equivalent. In the low temperature phase we have computed $\chi$ and $\mu_{2}$ by using eqs. (1922), respectively, in the following way: Up to a certain distance $R$ we have used $G(r)$ computed directly from the configurations that we have generated. Since the relative statistical error increases exponentially with the distance $r$, for $r>R$ we have used instead

$$
\tilde{G}(r)=G(R) \exp \left(-\frac{r-R}{\xi_{\text {eff }}(R)}\right) .
$$

In the following analysis we have used the data obtained by choosing $R \approx 4 \xi_{\text {eff }}(R)$. We have checked that these results are consistent with those obtained for $R \approx 3 \xi_{\text {eff }}(R)$.

\section{THE SIMULATIONS}

\section{A. The Monte Carlo algorithm}

Analogous to [21], we have simulated the Blume-Capel model using a hybrid of local updates and single cluster updates [22]. In the high temperature phase we have used as local update the heat-bath algorithm. With the local update we run through the lattice in typewriter fashion. Running through the lattice once is called one sweep in the following. After two heat-bath sweeps we perform a certain number $N_{c l}$ of single cluster updates. We have chosen $N_{c l}$ to be roughly one third of the number of lattice sites $V$ divided by the average size of a cluster. In the following we shall denote two heat-bath sweeps followed by $N_{c l}$ single cluster updates as one cycle of the update. In the high temperature phase we have used the cluster algorithm to compute improved estimators of the magnetic susceptibility and the second moment correlation length.

In the low temperature phase we have used a local Metropolis update that is implemented in multispin coding technique [23]. Details of our implementation can be found in [6]. Here, after ten sweeps of the local update, we performed $N_{c l}$ single cluster updates. Also here we have chosen $N_{c l}$ to be roughly one third of the number of lattice sites $V$ divided by the average size of a cluster. Here we denote ten sweeps of the local update followed by $N_{c l}$ single cluster updates as one cycle. In the low temperature phase we did not use cluster improved estimators since they do not reduce the statistical error significantly in this phase.

As random number generator we have used the SIMD-oriented Fast Mersenne Twister algorithm [24].

\section{B. Simulations in the high temperature phase}

First we have checked which lattice sizes are needed to keep the deviation from the thermodynamic limit smaller than the statistical error of the observables that we measure. 
Based on finite size scaling theory [25], we expect that the $L$-dependence of a singular observable $A$ is given by

$$
A(L, \beta)=A(\infty, \beta) \times\left[1+g_{A}(L / \xi(\beta))\right],
$$

where we have ignored corrections to scaling. In the absence of a massless mode, as it is the case here,

$$
g_{A}(L / \xi) \simeq c_{A} \exp (-L / \xi)
$$

for large values of $L / \xi$. At $D=0.655$ and $\beta=0.372$ we have simulated lattices with the linear lattice sizes $L=18,20,22,24,26,28,32,40$ and 48 to check the size dependence of the observables. In total these simulations took about 6 days of CPU time on single core of a Quad-Core Opteron(tm) 2378 CPU $(2.4$ GHz). Our results are summarized in table II. We give the number of update cycles (stat), the number of single cluster updates $N_{c l}$ per update cycle and the estimates of the energy density $E$, the magnetic susceptibility $\chi$ and the second moment correlation length $\xi_{2 n d}$. We have fitted these results with the ansatz $A(L)=A(\infty)+c_{A} \exp \left(-L / \xi_{\text {exp }}\right)$, where we have taken $\xi_{\text {exp }}=3.09394(13)$, which is the result for the exponential correlation length that we have obtained for $L=48$. Skipping the data for $L=18$ we get an acceptable $\chi^{2} /$ d.o.f. for all three quantities. The result for the thermodynamic limit $A(\infty)$ is given in the last row of table II. The correction amplitudes are $c_{E}=0.114(3), c_{\chi}=-21.2(1.1)$ and $c_{\xi_{2 n d}}=-1.28(8)$. This means that the deviation from the thermodynamic limit is of the same size as the statistical error that we have reached here for $L / \xi \approx 10$ for all three quantities that we have studied.

In the following simulations we have chosen $L \gtrsim 10 \xi_{2 n d}$ throughout. Since for most of our simulations $L$ is clearly larger than $10 \xi_{2 n d}$ and the relative statistical error of the magnetic susceptibility and the second moment correlation length is larger than that of the results discussed above, we expect that deviations from the thermodynamic limit can be safely ignored.

In the case of $D=0.655$ we have simulated at 201 different values of $\beta$ starting from $\beta=0.25$ up to $\beta=0.3872$. For each value of $\beta$ we have performed 500000 update cycles. At $\beta=0.3872$ we have simulated an $L=300$ lattice and find $\xi_{2 n d}=26.698(7)$. In the case of $D=0.641$ we have only simulated at 12 different values of $\beta$ from $\beta=0.3827$, where $\xi_{2 n d}=8.8993(7)$, up to $\beta=0.3849$, where $\xi_{2 n d}=20.859(3)$. Here, for the two smallest values of $\beta$ we performed about $3 \times 10^{6}$ update cycles and about $1.5 \times 10^{6}$ for the larger ones. The simulations in the high temperature phase at $D=0.655$ and 0.641 together took about one year of CPU time on a single core of a Quad-Core Opteron(tm) $2378 \mathrm{CPU}(2.4 \mathrm{GHz})$.

\section{Simulations in the low temperature phase}

Also here we have checked which lattice sizes are needed to keep deviations from the thermodynamic limit sufficiently small to be safely ignored. To this end, we performed simulations at $D=0.655$ and $\beta=0.405$ using the linear lattice sizes $L=12,16,20,24$, 30 and 40. These simulations took about three weeks of CPU time on a single core of a Quad-Core Opteron(tm) $2378 \mathrm{CPU}(2.4 \mathrm{GHz})$. Our results for the various observables are summarized in table II. Starting from $L=20$ the results are consistent among each other. 
TABLE I: The energy density $E$, the magnetic susceptibility $\chi$ and the second moment correlation length $\xi_{2 n d}$ at $D=0.655$ and $\beta=0.372$ for various linear lattice sizes $L$. Furthermore we give the number of update cycles (stat) and the number of single cluster updates $N_{c l}$ per update cycle. In the last raw we give the result of our extrapolation to the thermodynamic limit, as discussed in the text.

\begin{tabular}{|c|c|c|c|c|}
\hline$L$ & stat $/ 10^{6}$ & $\overline{N_{c l}}$ & $\chi$ & $\xi_{2 n d}$ \\
\hline 18 & 50 & $2000.492029(5)$ & $24.4851(18)$ & $3.07593(13)$ \\
\hline 20 & 40 & 3000 & & 3.07794 \\
\hline 22 & 35 & $4000.491721(4)$ & 24.5405 & $3.07910(12)$ \\
\hline 24 & 30.9 & $5000.491678(4)$ & 24.5469 & $3.07945(11)$ \\
\hline 26 & 32.4 & $700 \quad 0.491658(3)$ & $24.5502(13)$ & $3.07963(10)$ \\
\hline 28 & 20 & $10000.491643(4)$ & $24.5532(14)$ & $3.07985(11)$ \\
\hline 32 & 10 & $10000.491635(5)$ & $24.5538(18)$ & $3.08000(13)$ \\
\hline 40 & 7.5 & $25000.491633(4)$ & $24.5532(14)$ & $3.07994(11)$ \\
\hline 48 & 5 & $20000.491633(4)$ & $24.5545(15)$ & $3.08008(12)$ \\
\hline & & $0.4916314(17)$ & $24.5549(7)$ & $3.08000(5)$ \\
\hline
\end{tabular}

TABLE II: Estimates for the energy density $E$, the magnetization $m$, the magnetic susceptibility $\chi$, the second moment correlation length $\xi_{2 n d}$ and the exponential correlation length $\xi_{\text {exp }}$ at $D=0.655$ and $\beta=0.405$ for various linear lattice sizes $L$. The results for $\chi$ and $\xi_{2 n d}$ are obtained with $R=4$ and correspondingly, $\xi_{\text {exp }}$ is approximated by $\xi_{\text {eff }}(6)$, since $4 \xi_{\text {exp }} \approx 6$. Furthermore we give the number of update cycles (stat) that we have performed. This number includes the factor 64 of copies of the system that we have simulated. In all cases we have performed two single cluster updates for each update cycle.

\begin{tabular}{lrllccc}
\hline$L$ & stat $/ 10^{6}$ & $E$ & $m$ & $\chi$ & $\xi_{2 n d}$ & $\xi_{\exp }$ \\
\hline 12 & 640 & $1.063260(12)$ & $0.514811(5)$ & $4.4891(4)$ & $1.5483(3)$ & $1.6056(6)$ \\
16 & 320 & $1.063467(11)$ & $0.514997(5)$ & $4.4317(4)$ & $1.5271(3)$ & $1.5778(7)$ \\
20 & 320 & $1.063487(8)$ & $0.515010(3)$ & $4.4281(4)$ & $1.5258(3)$ & $1.5759(6)$ \\
24 & 190 & $1.063474(8)$ & $0.515005(3)$ & $4.4283(4)$ & $1.5259(4)$ & $1.5761(8)$ \\
30 & 130 & $1.063482(7)$ & $0.515008(3)$ & $4.4284(4)$ & $1.5258(4)$ & $1.5762(8)$ \\
40 & 32 & $1.063474(9)$ & $0.515004(4)$ & $4.4277(7)$ & $1.5243(7)$ & $1.5729(15)$ \\
\hline
\end{tabular}

Below we followed the recommendation of [8] and have used lattices with $L>20 \xi_{2 n d}$. Given the observations made here, this is surely a safe choice.

In the case of $D=0.655$ we have simulated 64 different values of $\beta$ starting from $\beta=$ 0.3884 , where $\xi_{2 n d}=11.687(45)$ up to $\beta=0.42$, where $\xi_{2 n d}=1.0293(7)$. In addition we have simulated at 85 values of $\beta$ up to $\beta=0.60$, where we have only measured the energy density. In the case of $D=0.641$ we have simulated at 12 different values of $\beta$ starting from $\beta=0.3866$, where $\xi_{2 n d}=9.589(19)$, up to $\beta=0.3899$, where $\xi_{2 n d}=4.3749(34)$. In total these simulations in the low temperature phase took about 10 years of CPU time 
on a single core of a Quad-Core Opteron(tm) $2378 \mathrm{CPU}(2.4 \mathrm{GHz})$.

\section{UNIVERSAL AMPLITUDE RATIOS}

First we have computed the ratio of the amplitudes of the magnetic susceptibility in the high and the low temperature phase. To this end one could fit the data for the magnetic susceptibility with an ansatz such as eq. (2) for the data in the high and the low temperature phase separately. Using the results for $C_{+}$and $C_{-}$obtained this way one could compute the ratio $C_{+} / C_{-}$. Instead, following ref. [8] we use a different strategy. The amplitude ratio can be defined as

$$
\frac{C_{+}}{C_{-}}=\lim _{t \searrow 0} \frac{\chi(t)}{\chi(-t)} .
$$

Following this definition, we have first calculated the the ratio $\chi(t) / \chi(-t)$ at finite values of $t=\beta_{c}-\beta$. To this end, we have computed for the values $\beta_{\text {low }}$, where we have simulated in the low temperature phase corresponding values $\beta_{\text {high }}=2 \beta_{c}-\beta_{\text {low }}$. Here we made no effort to simulate exactly at these values of $\beta_{h i g h}$. Instead we interpolate between the values that we have simulated. To this end, we take the $\beta_{-} \leq \beta_{\text {high }} \leq \beta_{+}$ which are closest to $\beta_{\text {high }}$. We compute $c\left(\beta_{ \pm}\right)=\chi\left(\beta_{ \pm}\right)\left(\beta_{c}-\beta_{ \pm}\right)^{\gamma}$, using $\gamma=1.23719$ [6]. Then we linearly interpolate to get an estimate of $c\left(\beta_{\text {high }}\right)$. Finally we compute $\chi\left(\beta_{\text {high }}\right)=c\left(\beta_{\text {high }}\right)\left(\beta_{c}-\beta_{\text {high }}\right)^{-\gamma}$. Since we have simulated a large number of $\beta$-values, the systematical error introduced by this interpolation should be negligible. In figure 1 we have plotted our results for $\chi(t) / \chi(-t)$ as a function of $t$. From RG theory we expect that

$$
\frac{\chi(t)}{\chi(-t)}=\frac{C_{+}}{C_{-}}+a t^{\theta}+b t+c t^{\theta^{\prime}}+d t^{2 \theta}+e t^{\gamma}+\ldots
$$

where $a$ and in particular $d$ should be small here, since $D=0.655$ is a good approximation of $D^{*}$. Following ref. [26] $\theta^{\prime}=1.05(7)$. Therefore the term $c t^{\theta^{\prime}}$ can be hardly discriminated from the analytic correction $b t$. The term $e t^{\gamma}$ is caused by the analytic background of the magnetic susceptibility. Figure 1 suggests that $\chi(t) / \chi(-t)$ is essentially a linear function of $t$. Therefore, the terms explicitly given in eq. (27) should be sufficient to fit $\chi(t) / \chi(-t)$. In particular we have fitted our data with the ansätze

$$
\begin{aligned}
& \frac{\chi(t)}{\chi(-t)}=\frac{C_{+}}{C_{-}}+b t \\
& \frac{\chi(t)}{\chi(-t)}=\frac{C_{+}}{C_{-}}+a t^{\theta}+b t \\
& \frac{\chi(t)}{\chi(-t)}=\frac{C_{+}}{C_{-}}+a t^{\theta}+b t+e t^{\gamma}
\end{aligned}
$$

using $\theta=0.524$ and $\gamma=1.23719$. Using these ansätze we have performed a large number of fits. Below we give the results of those fits that include a maximal number of data points under the condition that $\chi^{2} /$ d.o.f. is close to one. The fits are done using our data for $D=0.655$ if not stated otherwise. Fitting the data that satisfy $t<0.005$ with the ansatz (28) we get $C_{+} / C_{-}=4.7089(14), b=12.0(4)$ and $\chi^{2} /$ d.o.f. $=24.6 / 20$. Fitting all available data for $D=0.641$ with ansatz (28) we get $C_{+} / C_{-}=4.7145(21)$, 


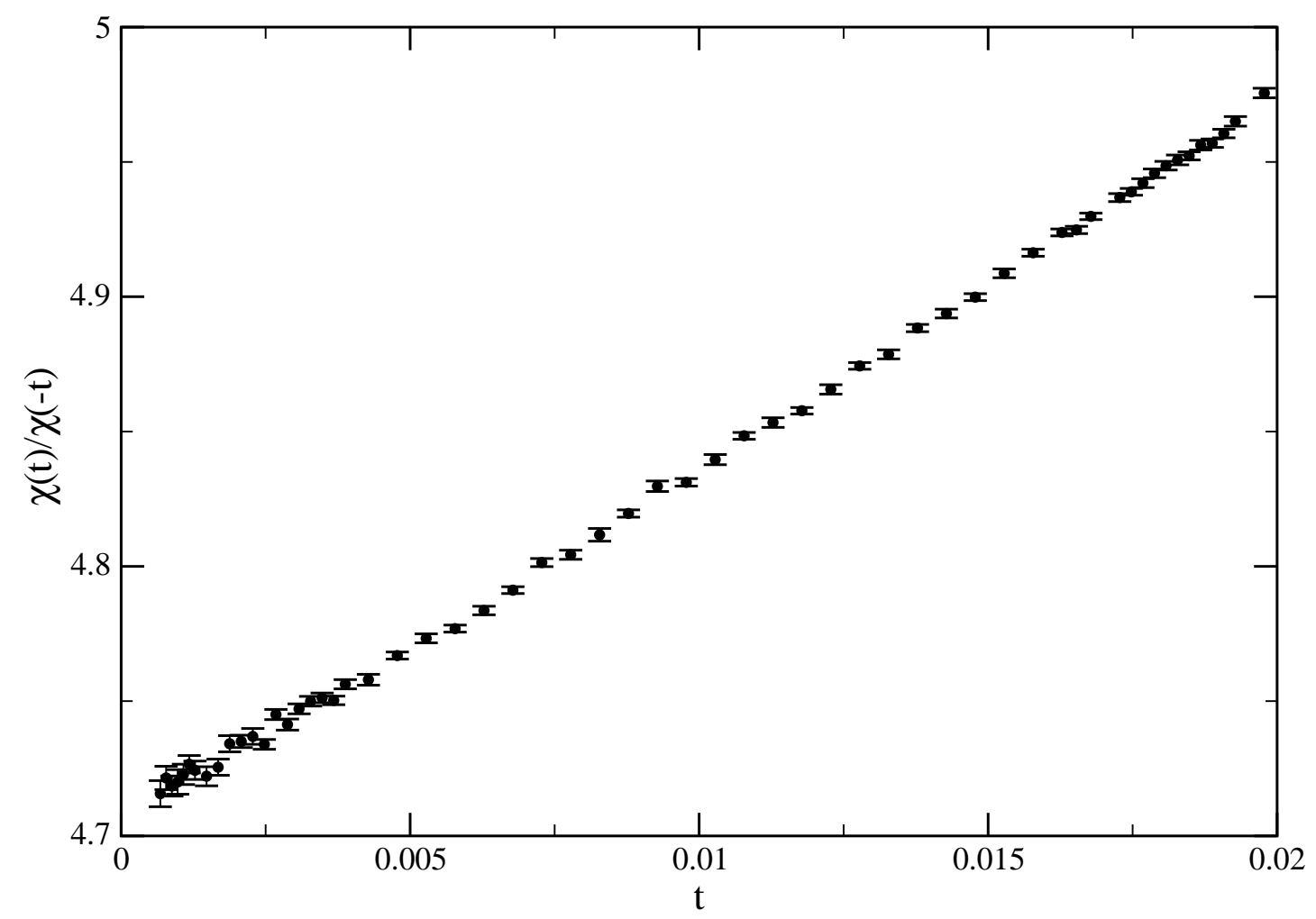

FIG. 1: We plot the ratio $\chi(t) / \chi(-t)$ as a function of $t=\beta_{c}-\beta$ computed from our data for $D=0.655$. For a discussion see the text.

$b=11.4(9)$ and $\chi^{2} /$ d.o.f. $=9.6 / 8$. Using ansatz (29), fitting data with $t<0.019$ we get $C_{+} / C_{-}=4.718(2), a=-0.43(6), b=15.6(3)$ and $\chi^{2} /$ d.o.f. $=55.8 / 53$. Fitting the data that satisfy $t<0.022$ with the ansatz (30) we get $C_{+} / C_{-}=4.712(6), a=-0.05(30)$, $b=8.7(4.9), e=12.3(8.2)$ and $\chi^{2} /$ d.o.f. $=66.6 / 57$. As our final result we quote

$$
\frac{C_{+}}{C_{-}}=4.713(7)
$$

which is chosen such that it covers all results, including their error bars, of the fits quoted above. We have estimated the error due to the uncertainty of $\beta_{c}$ by redoing some of the fits using ratios computed with $\beta_{\text {high }}=2\left(\beta_{c}+\right.$ error $)-\beta_{\text {low }}$. We find that it is clearly smaller than the error quoted above.

Next we have computed the amplitude ratio

$$
\frac{f_{2 n d,+}}{f_{2 n d,-}}=\lim _{t \searrow 0} \frac{\xi_{2 n d}(t)}{\xi_{2 n d}(-t)} .
$$

The calculation is analogous to that of the ratio $C_{+} / C_{-}$. Therefore we abstain from giving details and directly quote our final result

$$
\frac{f_{2 n d,+}}{f_{2 n d,-}}=1.939(5) .
$$




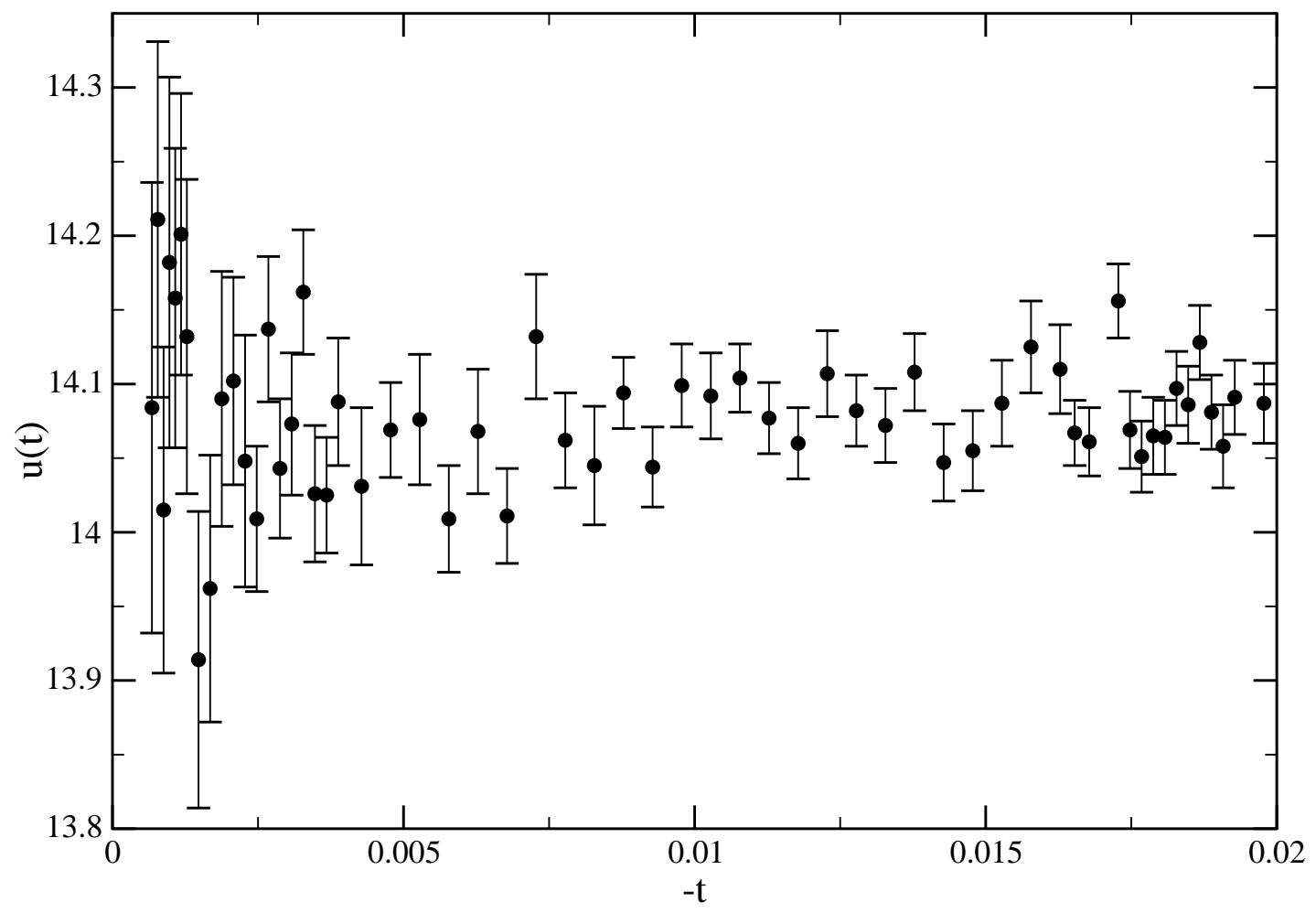

FIG. 2: We plot the ratio $u(t)=3 \chi(t) / \xi_{2 n d}^{3}(t) m^{2}(t)$ as a function of $-t=\beta-\beta_{c}$ computed from our data for $D=0.655$. For a discussion see the text.

Next we have computed the RG-invariant quantity

$$
Q_{c}=\frac{f_{2 n d,+}^{3} B^{2}}{C_{+}}
$$

where $B$ is the amplitude of the spontaneous magnetization in the low temperature phase. To this end, we have first evaluated $r=\xi_{2 n d}^{3} / \chi$ for all values of $\beta$ that we have simulated in the high temperature phase, where we have computed the statistical error of this combined quantity using the Jackknife method. Then we have computed

$$
Q_{c}=\lim _{t \searrow 0} r(t) m^{2}(-t)
$$

in the same fashion as we have computed $C_{+} / C_{-}$and $f_{2 n d,+} / f_{2 n d,-}$ above. As our final estimate we quote

$$
Q_{c}=0.3293(2) \text {. }
$$

The renormalized coupling in the low temperature phase is given by

$$
u^{*}=\frac{3 C_{-}}{f_{2 n d,-}^{3} B^{2}}=\lim _{t \nearrow 0} \frac{3 \chi(t)}{\xi_{2 n d}^{3}(t) m^{2}(t)} .
$$

In figure 2 we plot $3 \chi(t) / \xi_{2 n d}^{3}(t) m^{2}(t)$ as a function of $-t$. Since only quantities in the low temperature phase are involved, there should be no analytic correction. However, 
since $\theta^{\prime} \approx 1$ we kept a term bt in our ansätze. Based on various fits we arrive at the final estimate

$$
u^{*}=14.08(5) \text {. }
$$

Now let us consider the ratio $\xi_{\exp } / \xi_{2 n d}$. It turns out that it is difficult to determine the exponential correlation length accurately in the low temperature have. The time slice correlation function behaves as

$$
G(r)=c_{1} \exp \left(-r / \xi_{1}\right)+c_{2} \exp \left(-r / \xi_{2}\right)+\ldots
$$

Since the ratio $\xi_{1} / \xi_{2}=1.83(3)[27]$ is rather small, the effective correlation length $\xi_{\text {eff }}$, eq. (20), converges only rather slowly to $\xi_{\text {exp }}$. On the other hand, the relative statistical error of $G(r)$ increases exponentially. Therefore very large distances that are needed to get a small deviation of $\xi_{\text {eff }}$ from $\xi_{\text {exp }}$ are not accessible. As compromise, we have taken $\xi_{\text {eff }}(R)$ with $R \approx 4 \xi_{\text {eff }}(R)$ as our final estimate. To check the systematical error introduced this way, we have compared our result with that for $R \approx 3 \xi_{\text {eff }}(R)$. As our final estimate we quote

$$
\frac{f_{\exp ,-}}{f_{2 n d,-}}=1.020(5)
$$

Here the error should cover both the systematical deviation of $\xi_{\text {eff }}$ from $\xi_{\text {exp }}$ as well as systematical errors due to subleading corrections that are not included in our fits. In order to get more precise results for $\xi_{\text {exp }}$ and as a consequence for $f_{\text {exp },-} / f_{2 n d,-}$ a variational analysis of a large set of correlation functions, as it has been done in ref. [27] would be useful. Furthermore the method of [28] to reduce the variance of correlation functions could help to compute $G(r)$ accurately at large distances $r$.

\section{A. Ratios that involve the specific heat}

In order to compute amplitude ratios that involve the specific heat, we have analyzed our data for the energy density which can be accurately determined in the simulation. In the case of the energy density we have to separate the analytic background and the singular part, which is needed here. In the neighborhood of the critical point, the energy density behaves as

$$
E=E_{b}+E_{s}
$$

where the analytic background can be Taylor expanded around the critical point:

$$
E_{b}(\beta)=E_{n s}+C_{n s}\left(\beta-\beta_{c}\right)+d_{n s}\left(\beta-\beta_{c}\right)^{2}+\ldots .
$$

The singular part is given by

$$
E_{s}=a_{ \pm}|t|^{-\alpha} \times\left(1+b_{ \pm}|t|^{\theta}+c t+\ldots\right)
$$

In a first step we have analyzed data generated in relation with [6] for cubic systems with a linear size up to $L=360$ and periodic boundary conditions directly at the critical point. At the critical point the energy density behaves as

$$
E=E_{n s}+a L^{-3+1 / \nu}\left(1+c L^{-\omega}+\ldots\right)
$$


and the specific heat as

$$
C=C_{n s}+b L^{-3+2 / \nu}\left(1+d L^{-\omega}+\ldots\right)
$$

Here we perform fits fixing $\nu=0.63002(10)$ as obtained in [6]. Our final estimate is taken from fits without any correction term and all lattice sizes that are larger or equal to $L_{\text {min }}=24$ taken into account. Systematic errors are estimated by performing fits that include corrections with an exponent that is either $0.832,1.6$ or 2 . For $D=0.655$ we get

$$
E_{n s}=0.602111(1)+0.006 \times(\nu-0.63002)+42 \times\left(\beta_{c}-0.387721735\right)
$$

and

$$
a=1.7490(5)+14 \times(\nu-0.63002)-1800 \times\left(\beta_{c}-0.387721735\right) .
$$

We have redone the fits with slightly shifted values of the input parameters $\nu$ and $\beta_{c}$ to obtain the dependence of $E_{n s}$ and $a$ on these parameters. For the specific heat at $D=0.655$ we get

$$
C_{n s}=-19.1(1)-1700 \times(\nu-0.63002)-1300000 \times\left(\beta_{c}-0.387721735\right)
$$

and

$$
b=25.30(5)+1350 \times(\nu-0.63002)+620000 \times\left(\beta_{c}-0.387721735\right)
$$

from fits with $L_{\min }=64$. The error is dominated by systematical errors that we have estimated from fits that include corrections to scaling.

For the energy density at $D=0.641$ we get

$$
E_{n s}=0.604870(2)+0.01 \times(\nu-0.63002)+41 \times\left(\beta_{c}-0.38567122\right)
$$

and

$$
a=1.749(1)+14 \times(\nu-0.63002)-1800 \times\left(\beta_{c}-0.38567122\right) .
$$

For the specific heat at $D=0.641$ we get

$$
C_{n s}=-19.1(2)-1700 \times(\nu-0.63002)-1000000 \times\left(\beta_{c}-0.38567122\right)
$$

and

$$
b=25.3(1)+1350 \times(\nu-0.63002)+500000 \times\left(\beta_{c}-0.38567122\right) .
$$

Next we have analyzed our data for the thermodynamic limit in the neighborhood of the critical point using the ansatz

$$
E(\beta)=E_{n s}+C_{n s}\left(\beta-\beta_{c}\right)+a_{ \pm}\left|\beta-\beta_{c}\right|^{1-\alpha}+d_{n s}\left(\beta-\beta_{c}\right)^{2}+b_{ \pm}\left|\beta-\beta_{c}\right|^{2-\alpha},
$$

where $E_{n s}, C_{n s}$ obtained above and $\beta_{c}=0.387721735(25)$ and $\alpha=0.10994(30)$ [6] are input parameters, while $a_{ \pm}, d_{n s}$ and $b_{ \pm}$are the 5 free parameters of the fit. Using the results of these fits we have computed $A_{+} / A_{-}=-a_{+} / a_{-}$and $P=\left(1-A_{+} / A_{-}\right) / \alpha$, which depends less on the input value for $\alpha$ than $A_{+} / A_{-}$.

Fitting all data for $D=0.655$ in the interval $\left[\beta_{c}-0.004, \beta_{c}+0.004\right]$ we get $A_{+} / A_{-}=$ 0.53611(7), $P=4.2195(6)$ and $\chi^{2} /$ d.o.f. $=55.5 / 51$ using the central values of the input parameters. For the interval $\left[\beta_{c}-0.0075, \beta_{c}+0.0075\right]$ we get $A_{+} / A_{-}=0.53614(3)$, $P=4.2192(3)$ and $\chi^{2} /$ d.o.f. $=165.7 / 93$. As a check we have also fitted with an ansatz, 
where we have added a term $\propto\left(\beta-\beta_{c}\right)^{3}$ compared with the ansatz (54). The results for $A_{+} / A_{-}$and $P$ change little compared with those given above. It turns out that the error of $A_{+} / A_{-}$and $P$ is actually dominated by the error induced by the uncertainty of our input parameters, $E_{n s}, c_{n s}, \beta_{c}$ and $\alpha$. In order to estimate this error, we have repeated the fits using shifted values of these input parameters. For example, we have replaced $E_{n s}$ by $\left(E_{n s}+\right.$ error $)$.

In order to check for the effect of leading corrections to scaling, we have fitted all our data at $D=0.641$ using the ansatz (54). We find $A_{+} / A_{-}=0.53624(11), P=4.2183(10)$ and $\chi^{2} /$ d.o.f. $=22.3 / 19$ using the central values of the input parameters. This means that the results obtained at $D=0.641$ and $D=0.655$ are fully consistent.

We arrive at the final estimates

$$
\frac{A_{+}}{A_{-}}=0.536(2)
$$

where the error is dominated by the uncertainty of $\alpha$, followed by the uncertainty of $C_{n s}$. In contrast

$$
P=4.22(1)
$$

depends much less on the value of $\alpha$. Its error is dominated by the uncertainty of $C_{n s}$. This different behavior of $A_{+} / A_{-}$and $P$ is actually much more important in the case of the XY-universality class, where $\alpha$ is close to zero and therefore the relative accuracy of $\alpha$ is much smaller than in the present case.

In order to compute the quantities

$$
Q_{\xi,+}=\alpha A_{+} f_{2 n d,+}^{3}
$$

and

$$
Q_{\xi,-}=\alpha A_{-} f_{2 n d,-}^{3}
$$

we have approximated the singular part of the energy density by

$$
E_{s}(t)=E(t)-E_{n s}-C_{n s} t \text {. }
$$

Then we have computed

$$
q(t)=t \xi_{2 n d}^{3}(t) E_{s}(t)
$$

The quantity $Q_{ \pm}$is then given by

$$
\begin{aligned}
& Q_{+}=\alpha(1-\alpha) \lim _{t \searrow 0} q(t) \\
& Q_{-}=\alpha(1-\alpha) \lim _{t \nearrow 0} q(t) .
\end{aligned}
$$

We have fitted our data with the ansatz

$$
q(t)=q^{*}+a t
$$

In the high temperature phase we find by fitting all data with $t<0.006$ the result $q^{*}=0.19412(3)$. We have redone this analysis with shifted values of $C_{n s}$ and $E_{n s}$ to estimate the effect on our result for $q^{*}$. It turns out that the error is dominated by the errors induced by the uncertainty of $C_{n s}$ and $E_{n s}$. We have also redone the analysis using 
TABLE III: Results for universal amplitude ratios obtained by high and low temperature series expansions of three different improved lattice models [14] and Monte Carlo simulations of the spin-1/2 Ising model [8, 9, 13] and the improved $\phi^{4}$ model [12]. In all these cases a simple cubic lattice has been studied.

\begin{tabular}{lcccccccc}
\hline Ref. & $A_{+} / A_{-}$ & $C_{+} / C_{-}$ & $\frac{f_{2 n d,+}}{f_{2 n d,-}}$ & $\frac{f_{\text {exp },-}}{f_{2 n d,-}}$ & $Q_{+}$ & $Q_{-}$ & $u^{*}$ & $Q_{c}$ \\
\hline here & $0.536(2)$ & $4.713(7)$ & $1.939(5)$ & $1.020(5)$ & $0.01899(10)$ & $0.00487(2)$ & $14.08(5)$ & $0.3293(2)$ \\
{$[30]$} & & & & & & & $14.25(12)$ & \\
{$[14]$} & $0.532(3)$ & $4.76(2)$ & $1.956(7)$ & & $0.01880(8)$ & $0.00472(5)$ & $0.3315(10)$ \\
{$[8]$} & & $4.75(3)$ & $1.95(2)$ & $1.017(7)$ & & & $14.3(1)$ & $0.328(5)$ \\
{$[9]$} & $0.560(10)$ & & & & & & \\
{$[12]$} & & $4.756(28)$ & $1.935(14)$ & & & & & \\
{$[13]$} & $0.532(7)$ & & & & & & & \\
\hline
\end{tabular}

our data for $D=0.641$. We get an estimate for $q^{*}$ that is fully consistent with that for $D=0.655$. We arrive at the final result

$$
Q_{+}=\alpha(1-\alpha) q^{*}=0.01899(10)
$$

Performing a similar analysis we arrive at

$$
Q_{-}=0.00487(2)
$$

\section{COMPARISON WITH RESULTS GIVEN IN THE LITERATURE}

\section{A. Monte Carlo simulations and high and low temperature series}

In table III we confront our results with those of previous Monte Carlo simulations [8, 9, 12, 13], with a comprehensive analysis of high and low temperature series [14] and the low temperature series estimate of $u^{*}$ given in [30]. In [14] a parametric representation of the equation of state has been used to obtain results for the critical isotherm and the low temperature phase from high temperature series. For an exhaustive overview of the literature see table 11 of [4].

In [8, 9] we have simulated the spin-1/2 Ising model on simple cubic lattices of a linear size up to $L=120$ and $L=128$, respectively. Also the authors of [13] have simulated the spin-1/2 Ising model on the simple cubic lattice. They have simulated at a large number of $\beta$-values in both phases of the model on lattices of a size up to $L=128$. In [12] the $\phi^{4}$ model on the simple cubic lattice has been simulated at $\lambda=1.1$, which is the estimate of $\lambda^{*}$ obtained in ref. [29]. The authors have simulated lattices up to the size $L=120$. In addition to simulations at a vanishing external field $h=0$, they have simulated $h \neq 0$ at the critical temperature. This allowed them to compute additional universal amplitudes ratios that we do not discuss here.

Essentially our results confirm those of the previous work. Even in the worst case, the deviation between our result and that of the other works summarized in table III is less than three times the combined error. 
TABLE IV: Results for universal amplitude ratios obtained by using the $\epsilon$-expansion $(\epsilon)$ and perturbation theory in three dimensions (3D) fixed. For the definition of the amplitude ratios and a discussion see the text.

\begin{tabular}{|c|c|c|c|c|c|c|c|}
\hline Ref. & Method & $A_{+} / A_{-}$ & $C_{+} / C_{-}$ & $\frac{f_{2 n d,+}}{f_{2 n d,-}}$ & $Q_{+}$ & $u^{*}$ & $Q_{c}$ \\
\hline [31] & $\epsilon$ & & 4.8 & 1.91 & & & \\
\hline$[32]$ & $\epsilon$ & 0.55 & 4.8 & & & & \\
\hline$[36]$ & $\epsilon$ & & & & $0.01966(17)$ & & \\
\hline [33] & $\epsilon$ & 0.44 & 4.9 & & 0.0223 & & \\
\hline 34$]$ & $\epsilon$ & $0.524(10)$ & & & & & \\
\hline$[35]$ & $\epsilon$ & $0.527(37)$ & $4.73(16)$ & & & & \\
\hline [39] & $3 \mathrm{D}$ & $0.541(14)$ & $4.77(30)$ & & & & $0.331(9)$ \\
\hline$[37]$ & $3 \mathrm{D}$ & & & & $0.01968(15)$ & & \\
\hline$[40]$ & $3 \mathrm{D}$ & $0.540(11)$ & & & & & \\
\hline [38] & $3 \mathrm{D}$ & & $4.72(17)$ & $2.013(28)$ & & 14.2 & \\
\hline$[35]$ & $3 \mathrm{D}$ & $0.537(19)$ & $4.79(10)$ & & & & \\
\hline 41$]$ & $3 \mathrm{D}$ & & & & 0.0203 & & \\
\hline
\end{tabular}

\section{B. Field theoretic methods}

In tableIV we have summarized results obtained from the $\epsilon$-expansion and perturbation theory in three dimensions fixed. Mostly we have taken these results from table 12 of ref. [4]. Note that in ref. [35] the field theoretic methods have been used in connection with a parametric representation of the equation of state. In ref. [39] $A_{+} C_{+} / B^{2}=0.0594(11)$ is given. By using the value of $Q_{+}$given e.g. by [37] $Q_{c}$ can be computed. Here we only report those amplitude ratios that we have computed in this work. For a comprehensive list of amplitude ratios see table 12 of ref. [4]. Essentially the field theoretic results are consistent ours, albeit their accuracy is clearly lower than ours. The errors for $Q_{+}$given by [36, 37] seems to be underestimated.

\section{Experiments}

Here we just mention the results of two experimental works to give the reader an idea of the accuracy that can be reached. Studying a mixture of succinonitrile and water the authors of [42] found $A_{+} / A_{-}=0.536 \pm 0.005$ and $Q_{+}=0.0187 \pm 0.0013$. Studying the antiferromagnet $\mathrm{FeF}_{2}$, the authors of [43] found $A_{+} / A_{-}=0.53 \pm 0.01$ and $C_{+} / C_{-}=$ $4.6 \pm 0.02$. In particular for $A_{+} / A_{-}$the accuracy of the experimental studies is close to ours. The results of both studies are consistent with ours, confirming universality. For a comprehensive summary of experimental results see refs. [4, 5]. 


\section{SUMMARY AND CONCLUSIONS}

We have simulated the Blume-Capel model on the simple cubic lattice at $D=0.641$ and 0.655 for a large number of inverse temperatures $\beta$ in a neighborhood of the critical point. These values of $D$ are close to $D^{*}=0.656(20)$, where the amplitudes of leading corrections to scaling vanish. We have simulated lattices up to $300^{3}$ in the high temperature and $500 \times 250^{2}$ in the low temperature phase. Throughout we have chosen the linear size $L$ of the lattice such that $L \gtrsim 10 \xi_{2 n d}$ in the high temperature and $L \gtrsim 20 \xi_{2 n d}$ in the low temperature phase to avoid significant deviations from the thermodynamic limit. In the high temperature phase at $D=0.655$ we have reached the correlation length $\xi_{2 n d}=26.698(7)$. Using the data obtained in these simulations we have extracted precise numerical estimates for a number of universal amplitude ratios. We carefully estimated systematical errors caused by subleading corrections.

In table III we have summarized our results and compare them with previous estimates obtained from Monte Carlo simulations or from high and low temperature series expansions of lattice models. Our results are essentially consistent with but more precise than previous estimates. The same holds for the comparison with field theoretic methods. Also the accuracy of experimental results given in the literature is lower than ours.

\section{ACKNOWLEDGEMENTS}

This work was supported by the DFG under the grant No HA 3150/2-1.

[1] K. G. Wilson and J. Kogut, Phys. Rep. C 12, 75 (1974).

[2] M. E. Fisher, Rev. Mod. Phys. 46, 597 (1974).

[3] M. E. Fisher, Rev. Mod. Phys. 70, 653 (1998).

[4] A. Pelissetto and E. Vicari, Phys. Rept. 368, 549 (2002) arXiv:cond-mat/0012164

[5] A. Aharony, P. C. Hohenberg and V. Privman, Universal critical point amplitude relations in "Phase transitions and Critical phenomena" vol.14, C.Domb and J.L. Lebowitz eds. (Academic Press 1991)

[6] M. Hasenbusch, Phys. Rev. B 82, 174433 (2010) arXiv:1004.4486

[7] J. H. Chen, M. E. Fisher, and B. G. Nickel, Phys. Rev. Lett. 48630 (1982); M. E. Fisher and J. H. Chen, J. Physique (Paris) 46, 1645 (1985).

[8] M. Caselle and M. Hasenbusch, J. Phys. A 30, 4963 (1997) arXiv:hep-lat/9701007

[9] M. Hasenbusch and K. Pinn, J. Phys. A 316157 (1998) arXiv:cond-mat/9706003

[10] M. Hasenbusch, J. Stat. Mech.: Theory Exp. 2006, P08019 arXiv:cond-mat/0607189

[11] M. Hasenbusch, J. Stat. Mech.: Theory Exp. 2008, P12006 arXiv:0810.2716]

[12] J. Engels, L. Fromme and M. Seniuch, Nucl. Phys. B 655, 277 (2003) arXiv:cond-mat/0209492

[13] X. Feng and H. W. J. Blöte, Phys. Rev. E 81, 031103 (2010).

[14] M. Campostrini, A. Pelissetto, P. Rossi and E. Vicari, Phys. Rev. E 65, 066127 (2002) arXiv:cond-mat/0201180].

[15] M. Deserno, Phys. Rev. E 56, 5204 (1997).

[16] J. R. Heringa and H. W. J. Blöte, Phys. Rev. E 574976 (1998). 
[17] Y. Deng and H. W. J. Blöte, Phys. Rev. E 70, 046111 (2004).

[18] M. Hasenbusch, Int. J. Mod. Phys. C 12, 991 (2001).

[19] K. Binder and H. Rauch, Z. Phys. 219, 201 (1969).

[20] K. Binder, J. Comp. Phys. 59, 1 (1985).

[21] R. C. Brower and P. Tamayo, Phys. Rev. Lett. 621087 (1989).

[22] U. Wolff, Phys. Rev. Lett. 62361 (1989).

[23] See, e.g., S. Wansleben, J. B. Zabolitzky, and C. Kalle, J. Stat. Phys. 37, 271 (1984); G. Bhanot, D. Duke, and R. Salvador, Phys. Rev. B 33, 7841 (1986).

[24] M. Saito and M. Matsumoto, "SIMD-oriented Fast Mersenne Twister: a 128bit Pseudorandom Number Generator", in Monte Carlo and Quasi-Monte Carlo Methods 2006, edited by A. Keller, S. Heinrich, H. Niederreiter, (Springer, 2008); M. Saito, Masters thesis, Math. Dept., Graduate School of schience, Hiroshima University, 2007. The source code of the program is provided at 'http://www.math.sci.hiroshima-u.ac.jp/ m-mat/MT/SFMT/index.html,

[25] M. N. Barber Finite-size Scaling in Phase Transitions and Critical Phenomena, Vol. 8, eds. C. Domb and J. L. Lebowitz, (Academic Press, 1983)

[26] K. E. Newman and E. K. Riedel, Phys. Rev. B 30, 6615 (1984).

[27] M. Caselle, M. Hasenbusch, P. Provero, Nucl. Phys. B 556, 575 (1999) arXiv:hep-lat/9903011

[28] M. Lüscher and P. Weisz, JHEP 0109, 010 (2001) arXiv:hep-lat/0108014

[29] M. Hasenbusch, J. Phys. A 32, 4851 (1999) arXiv:hep-lat/9902026

[30] A. Pelissetto and E. Vicari, Nucl. Phys. B 519, 626 (1998) arXiv:cond-mat/9711078

[31] E. Brézin, J. C. Le Guillou, and J. Zinn-Justin, Phys. Lett. A 47, 285 (1974).

[32] A. Aharony and P. C. Hohenberg, Phys. Rev. B 13, 3081 (1976).

[33] J. F. Nicoll and P. C. Albright, Phys. Rev. B 31, 4576 (1985).

[34] C. Bervillier, Phys. Rev. B 34, 8141 (1986).

[35] R. Guida and J. Zinn-Justin, J. Phys. A 31, 8103 (1998) arXiv:cond-mat/9803240

[36] C. Bervillier and C. Godrèche, Phys. Rev. B 21, 5427 (1980).

[37] C. Bagnuls and C. Bervillier, Phys. Rev. B 32, 7209 (1985).

[38] C. Gutsfeld, J. Küster, and G. Münster, Nucl. Phys. B 479654 (1996). arXiv:cond-mat/9606091

[39] C. Bagnuls, C. Bervillier, D. I. Meiron, and B. G. Nickel, Phys. Rev. B 35, 3585 (1987); (E) B 65, 149901 (2002)

[40] S. A. Larin, M. Mönnigmann, M. Strösser, and V. Dohm, Phys. Rev. B 58, 3394 (1998) arXiv:cond-mat/9805028

[41] M. Strösser and V. Dohm, Phys. Rev. E 67, 056115 (2003).

[42] A. W. Nowicki, M. Ghosh, S. M. McClellan, and D. T. Jacobs, J. Chem. Phys. 114, 4625 (2001).

[43] D. P. Belanger and H. Yoshizawa, Phys. Rev. B 35, 4823 (1987). 